\title{
Psychobiological Correlates of Aggression in Female Adolescents with Borderline Personality Disorder
}

\author{
Marialuisa Cavelti ${ }^{a} \quad$ Lena Rinnewitz $^{b} \quad$ Moritz Walter $^{b}$ Patrice van der Venne ${ }^{b}$ \\ Peter Parzer $^{c}$ Johannes Josi ${ }^{a}$ Katja Bertsch ${ }^{d, e}$ Romuald Brunner $^{f}$ \\ Franz Resch ${ }^{c}$ Julian Koenig ${ }^{a, g}, h \quad$ Michael Kaess ${ }^{a, b}$ \\ aUniversity Hospital for Child and Adolescent Psychiatry and Psychotherapy, University of Bern, Bern, Switzerland; \\ ${ }^{b}$ Department of Child and Adolescent Psychiatry, Section for Translational Psychobiology in Child and Adolescent \\ Psychiatry, Center for Psychosocial Medicine, University of Heidelberg, Heidelberg, Germany; ' ${ }^{\mathrm{D}}$ Department of Child \\ and Adolescent Psychiatry, Center for Psychosocial Medicine, Medical Faculty, University of Heidelberg, Heidelberg,

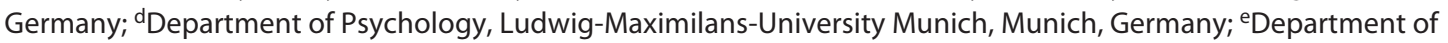 \\ Psychiatry, Center for Psychosocial Medicine, University of Heidelberg, Heidelberg, Germany; ${ }^{\text {Depepartment }}$ of Child \\ and Adolescent Psychiatry, University of Regensburg, Regensburg, Germany; ${ }^{9}$ Department of Child and Adolescent \\ Psychiatry, Psychosomatics and Psychotherapy, Faculty of Medicine and University Hospital Cologne, University of

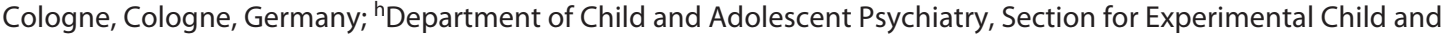 \\ Adolescent Psychiatry, Center for Psychosocial Medicine, University of Heidelberg, Heidelberg, Germany
}

\section{Keywords}

Adolescence - Borderline personality disorder - Aggression · Cortisol · Heart rate · Taylor Aggression Paradigm

\footnotetext{
Abstract

Introduction: Aggressive behavior in reaction to threats, frustration, or provocation is prevalent in borderline personality disorder (BPD). This study investigated aggressive behavior and its biological correlates in adolescents with BPD. Methods: Twenty-one female adolescents with a DSM-IV BPD diagnosis and 25 sex- and age-matched healthy controls participated in the Taylor Aggression Paradigm (TAP), a laboratory-based experiment measuring aggressive behavior in the interpersonal context. Heart rate was measured and saliva samples were taken throughout the experiment. Results: Multilevel mixed-effects linear regression analyses
}

karger@karger.com www.karger.com/psp

Karger $\stackrel{\text { ' }}{5}$

GOPEN ACCESS
(C) 2021 The Author(s)

Published by S. Karger AG, Basel

This is an Open Access article licensed under the Creative Commons Attribution-NonCommercial-4.0 International License (CC BY-NC) (http://www.karger.com/Services/OpenAccessLicense), applicable to the online version of the article only. Usage and distribution for commercial purposes requires written permission. revealed no significant group difference in aggressive behavior induced by the TAP. Additionally, the two groups did not differ in cortisol, testosterone, and heart rate responses to the aggression induction. The BPD group showed a significant cortisol increase in the time preceding the start of the TAP in contrast to the healthy control group, in whom a significant heart rate increase from baseline to the first block of the TAP was observed. Discussion: There was no evidence, either at the phenomenological or the biological level, of increased task-induced aggression in adolescents with $B P D$. The results may indicate that adolescents with BPD experienced fearful stress in anticipation of the experimental task in contrast to healthy controls who showed an adaptive response of the autonomic nervous system necessary to deal with the upcoming demand.

(c) 2021 The Author(s) Published by S. Karger AG, Basel
Correspondence to:

Michael Kaess, michael.kaess@upd.ch 


\section{Introduction}

Borderline personality disorder (BPD) is a severe mental disorder that is characterized by interpersonal instability, cognitive and self-disturbance, and affective and behavioral dysregulation [1]. It usually emerges during adolescence and early adulthood, affects $1-3 \%$ of adolescents in the general population, and can be reliably diagnosed during this developmental period [2]. Aggression, defined as any behavior directed toward others carried out with the intention to harm [3], is prevalent among people with BPD. Observational and experimental studies indicate that individuals with $\mathrm{BPD}$ engage in reactive (as opposed to instrumental) aggression [4-7], which occurs in the face of threats, frustration, or provocation [8]. Emotion regulation difficulties, poor impulse control (particularly when anger is experienced), threat hypersensitivity in social situations, and reduced cognitive empathy (i.e., the capacity to infer mental states of others) have been proposed as underlying mechanisms, linking BPD with aggressive behavior toward others [8-11]. Aggressive behavior makes individuals with BPD more likely to become involved with police and justice services, which significantly contributes to the high societal costs of the disorder [12]. Additionally, the high police and justice system utilization can negatively affect the chance of individuals with BPD to succeed in life (e.g., increased barriers to employment, housing, or financial aid, or loss of social support, stigma, and social exclusion) [13], which seems particularly relevant for young people. Notably, while the majority of studies examining aggressive behavior in BPD has been conducted in adults, a few studies also included young people (i.e., adolescents and young adults) [14-17]. For instance, in a data linkage study on 492 outpatients aged 15-25 years, DSM-IV BPD diagnosis and the number of BPD criteria were both found to predict an elevated risk for interpersonal violence. Interestingly, the impulsivity and anger criterion both independently predicted the risk for violent behavior [17], indicating that due to the heterogeneity of the disorder, not all affected individuals with BPD pathology may be at the same risk for aggressive behavior.

Little is known about the biological mechanisms underlying aggressive behavior in adolescents in general and in those with BPD pathology in particular. Biological stress regulation systems, including the endocrine and the autonomic nervous systems (ANS), have been linked to aggressive behavior. Although somewhat inconsistent, there is evidence suggesting that lower cortisol levels and heightened testosterone levels are associated with aggres- sive and antisocial behavior in adolescence [18-25]. It has been suggested that low cortisol is indicative of less fear, increasing the likelihood of aggressive behavior [26]. Testosterone plays a crucial role in the regulation of social behaviors, such as aggression, competition, and dominance [19], and the relationship between testosterone and aggression becomes stronger in transition into adolescence [25]. Additionally, low heart rate (HR) during resting-state and in response to stressors has been found to be a robust correlate of aggressive behavior in adolescents [27-31]. It has been argued that low arousal - as indicated by low HR - represents an unpleasant physiological state that leads to sensation-seeking behavior, including aggressive behavior [27, 32, 33]. Data on cortisol, testosterone, and HR in individuals with BPD are scarce and somewhat inconsistent. A recent meta-analysis by Thomas et al. [34] including 12 studies found significantly lower basal cortisol levels in adults with BPD compared to nonpsychiatric controls. In contrast, the meta-analysis by Drews et al. [35] including 37 studies revealed that adults with $\mathrm{BPD}$, when compared with healthy and clinical controls, demonstrate an elevated continuous cortisol secretion along with an attenuated cortisol response to psychosocial stress. Additionally, adults with BPD have been found to show elevated testosterone levels when compared with healthy controls [36-39]. Further, there is evidence for greater resting-state HR and reduced restingstate vagally mediated $H R$ variability (HRV) in adults and adolescents with BPD pathology [40-43]. To the best of our knowledge, no study today has investigated cortisol, testosterone, or HR in relation to aggressive behavior in adolescents with BPD.

In order to close this gap, the aim of the current study was to examine aggressive behavior and its biological correlates in adolescents with BPD compared to healthy controls. This was done using the Taylor Aggression Paradigm (TAP), a laboratory-based experiment measuring aggressive behavior in the interpersonal context [44]. The TAP has been successfully employed to examine the biological correlates of aggressive behavior in healthy, predominantly adult and gender-mixed samples [45-49]. Recently, Kogan-Goloborodko and colleagues [7] employed the TAP for the first time to a sample of adult BPD patients. Compared to healthy controls, the BPD patients showed higher overall aggression in the TAP, suggesting that this task is a useful measure of reactive aggression in this group. Rinnewitz et al. [50] have recently demonstrated that the TAP can successfully induce aggressive behavior and trigger increases in $\mathrm{HR}$ and cortisol in healthy female adolescents. No effects were observed on
Cavelti et al. 


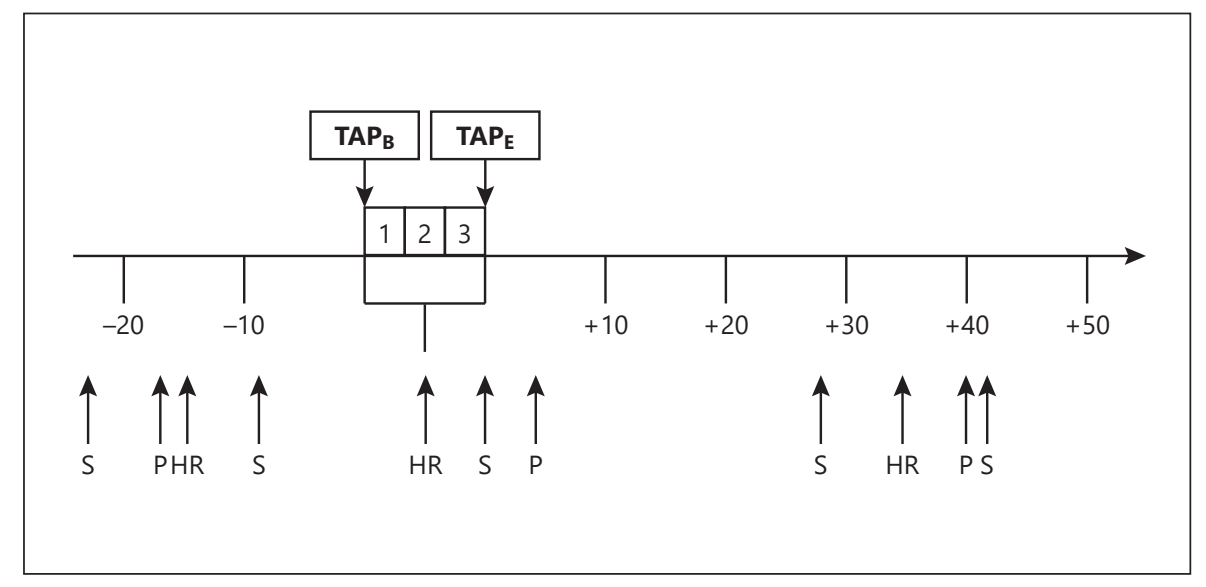

Fig. 1. After entering the experimental room, participants were attached with the HR monitor. HR was continuously recorded throughout the experiment. First, participants provided a saliva sample (T0, -23 min $\mathrm{TAP}_{\mathrm{B}}$ ) and completed the PANAS [55], followed by a second saliva sample $\left(\mathrm{T} 1,-9\right.$ min $\left.\mathrm{TAP}_{\mathrm{B}}\right)$. Next, participants conducted the TAP, while the experimenter left the room. Immediately after the TAP, participants provided a third saliva sample $\left(\mathrm{T} 2,+0\right.$ min $\left.\mathrm{TAP}_{\mathrm{E}}\right)$ and completed the PANAS a second time. Subsequently, participants completed the AQ [56], the BIS

the testosterone response to the TAP. Based on these findings, in the current study we applied the TAP to female adolescents with BPD and healthy controls in order to examine group differences in aggressive behavior and endocrinological and autonomic responses. We expected adolescents with BPD to demonstrate more aggressive behavior in the TAP compared with healthy controls. In addition, we assumed that adolescents with BPD would show attenuated cortisol and HR reactivity as well as increased testosterone reactivity to the TAP compared with healthy controls.

\section{Materials and Methods}

\section{Participants}

Participants were female adolescents between 13 and 17 years of age who were either diagnosed with BPD or were considered as mentally healthy. Adolescents with BPD were recruited from AtR!Sk ("Ambulanz für Risikoverhalten und Selbstschädigung”), a specialized outpatient service for adolescents presenting with BPD features at the Department of Child and Adolescent Psychiatry, University Hospital Heidelberg, Germany [51]. The service provides low-threshold initial contact, detailed and comprehensive diagnostic assessment of BPD features, and evidence-based therapeutic intervention for adolescents with any type of risk-taking or self-harming behavior (e.g., repeated nonsuicidal self-injury, suicide attempts, binge drinking, substance misuse, excessive gaming and internet use, risky sexual behavior, impulsive and delinquent behavior) [51]. Exclusion criteria for the BPD group were
11 [57], and the YPI [58]. Participants were further requested to complete a questionnaire assessing the credibility of the cover story. After that, participants provided a fourth saliva sample $(\mathrm{T} 3,+28$ min $\left.\mathrm{TAP}_{\mathrm{E}}\right)$. Finally, participants completed the PANAS a third time and provided a final saliva sample $\left(\mathrm{T} 4,+41\right.$ min $\left.\mathrm{TAP}_{\mathrm{E}}\right) . \mathrm{HR}$, Heart rate; PANAS, Positive And Negative Affect Schedule; TAP, Taylor Aggression Paradigm; AQ, Aggression Questionnaire; BIS, Barratt Impulsiveness Scale; YPI, Youth Psychopathic Traits Inventory.

an acute psychosis or acute suicidal crisis. Healthy controls were recruited from the general population via flyers and public advertisement. They were excluded from the study if they had a current psychiatric diagnosis, any psychological or psychiatric treatment in the last 2 years, or a history of nonsuicidal self-injury or suicidality. Participants from both groups were excluded if they had an irregular menstrual cycle or were pregnant, reported an endocrinological or neurological disorder, or took a glucocorticoid medication.

A total of 305 adolescents were screened for the study. Seventyseven adolescents were included in the study. Eleven participants dropped out during the study. The final sample consisted of $n=46$ participants; $n=21$ with BPD and $n=25$ healthy controls. The sample partly overlapped with the "aggression group" described in Rinnewitz et al. [50]. The 20 adolescents of the "control group" described in Rinnewitz et al. [50] were excluded from the current study.

\section{Procedure}

The study was approved by the Ethics Committee of the Medical Faculty at the University of Heidelberg (Study-ID: S-630/2013) and was conducted in accordance with the declaration of Helsinki [52]. Written informed consent was obtained from all participants and their parents or legal guardians. The study comprised 2 appointments of approximately $90 \mathrm{~min}$ each. At the first appointment, participants provided sociodemographic information and underwent a comprehensive diagnostic assessment, conducted by trained psychologists, in order to establish eligibility and assess psychopathology. The second appointment, the experimental session, was appointed individually for each participant to ensure that hormone samples were collected during the follicular phase of menstrual cycles. To limit the influence of diurnal variation on 
hormonal levels, experimental sessions were scheduled in the afternoon between 3:00 and 6:00 p.m. Participants were instructed to refrain from drinking and eating for at least half an hour and from physical exercise and smoking for two hours before the experiment. At arrival, participants were introduced to their female opponent who was an instructed actor. Participants and opponents were seated for approximately five minutes in the waiting area. They were told that they are going to play a competitive reaction time task in separated rooms to avoid reciprocal influence due to facial expression and gestures. Afterward, the experimental session, including the TAP, HR recoding, saliva sampling, and the assessment of affective changes and trait aggression and impulsivity was conducted as described in detail in Rinnewitz et al. [50]. Figure 1 provides an overview of the experimental session. At the end of the experimental session, participants were debriefed and received a compensation of 50 EUR for their time and expenses.

\section{Measures}

Clinical Interviews and Questionnaires

Sociodemographic data obtained included age, first language, education, body mass index, physical illness, oral contraceptives, smoking, and physical exercise. Eligibility of participants for the healthy control group was confirmed using the Structured Clinical Interview for DSM-IV Axis I/Non-Patient Edition (SCID-I/NP) [53], and for the BPD group using the BPD module of the Structured Clinical Interview for DSM-IV Axis II (SCID-II) [54].

Changes in affective states induced by the TAP were assessed using the Positive and Negative Affect Schedule (PANAS) [55], a self-report measure of positive affect (PA) and negative affect (NA). It consists of 20 items, each rated on a 5-point scale, ranging from 1 = "very slightly or not at all" to 5 = "extremely".

The Aggression Questionnaire (AQ) [56], the Barratt Impulsiveness Scale (BIS-11) [57], and the Youth Psychopathic Traits Inventory (YPI) [58] were used to examine differences in trait aggression and impulsivity between groups and for the purpose of comparison with state aggression measured by the TAP. The AQ is a self-report questionnaire assessing four dispositional sub-traits of aggression: anger, physical aggression, verbal aggression, and hostility. It comprises 29 items that are rated on a 5-point scale from $1=$ "extremely uncharacteristic of me" to 5 = "extremely characteristic of me". The BIS-11 is a 30 -item self-report questionnaire, assessing three aspects of impulsiveness: nonplanning, motor impulsivity, and attentional impulsivity. Items are scored on a 4-point scale from $1=$ "rarely/never" to 4 = "almost always/always". The YPI is a 50item self-report questionnaire, designed to assess three psychopathic-like traits in adolescents: grandiose-manipulative, callous-unemotional, and impulsive-irresponsible. It uses a 4-point scale ranging from $0=$ "does not apply at all" to $4=$ "applies very well".

\section{Taylor Aggression Paradigm}

A detailed description of the TAP is given in Rinnewitz et al. [50]. In brief, the TAP procedure was adopted from Böhnke and colleagues $[45,46]$, and E-Prime ${ }^{\circledR} 2.0$ [59] was used to present the experimental task on a laptop. The task consisted of 30 trials divided into three blocks of ten trials each. Participants were made to believe that they would play a reaction time task against an opponent (i.e., they had to press a key as quickly as possible, if a green square appeared) and that the loser of each trial would be punished by the winner with a blast of noise presented through headphones. Before each trial, participants were asked to select the volume and duration of the noise to be presented to their opponent in case she/ he loses. The ratio of win and lose trials was pre-programmed (i.e., each participant won and lost half of the trials) as were the noise volumes and durations (i.e., steady increase from block 1 to block $3)$. For each participant, the duration and volume settings were first averaged for each trial and then used as dependent variables in the statistical analyses.

\section{Endocrinological Measure}

Endocrinological reactivity to the TAP was measured using salivary cortisol and testosterone. Saliva samples were taken twice prior to and three times following the TAP (see Fig. 1). Saliva for cortisol analysis was collected using Salivette sampling devices (Sarstedt, Numbrecht, Germany). Saliva for testosterone analysis was sampled in SafeSeal micro tubes (Sarstedtx), because collection with the Salivette cotton swabs may introduce artifacts in the analyses of testosterone [60]. They were stored uncentrifuged at $-25^{\circ} \mathrm{C}$ until assay. Cortisol and testosterone levels were assayed at the Department of Psychology of the Dresden University of Technology, Germany, by using a luminsescence immunoassay, with a lower limit of detection of $0.276 \mathrm{nmol} / \mathrm{L}$. Mean and intra- and inter-assay coefficients of variation were $8 \%$.

\section{Autonomic Measure}

HR was recorded as a marker of autonomic reactivity to the TAP. It was continuously recorded during the experimental session with a Polar ${ }^{\circledR}$ RS800CX HR monitor (Polar Electro Oy, Kempele, Finland), while participants were seated. A strap with electrodes placed to the chest of participants sent wirelessly HR data to the monitor. HR was stored in 5-sec intervals and Polar ${ }^{\circledR}$ ProTrainer 5 (Polar Electro Oy) was used to transfer recordings onto a personal computer. Analysis of HR included five segments of five minutes each, before and after the TAP during resting conditions as well as during the three blocks of the TAP.

\section{Statistical Analyses}

Sociodemographic and clinical characteristics of the BPD group and the healthy control group were compared with twosample $t$ tests (with equal or unequal variances) for continuous variables and $\chi^{2}$ tests for categorical variables. Effect sizes were reported (Cohen's $d$ and Cramer's $V$ ).

Multilevel mixed-effects linear regression analyses were applied to examine differences by group over time in aggressive behavior assessed by the TAP, PA, and NA assessed by the PANAS, cortisol, testosterone, and HR. Fixed effects were GROUP, BLOCK of the TAP (for aggressive behavior and HR), and TIME (minutes for PA, $\mathrm{NA}$, cortisol, and testosterone) as well as their interactions. In all regression analyses, the subject ID was used as a random effect. The inspection of boxplots indicated the presence of three outliers concerning PA, five outliers concerning NA, two outliers concerning cortisol, five outliers concerning testosterone, and one outlier concerning HR. Sensitivity analyses revealed that the exclusion of these outliers did not change the results. Thus, outliers were retained in the final regression analyses presented below. In addition, the regression analyses were repeated, adjusting for smoking status, because the BPD group and the healthy control group significantly differed in the smoking status, as reported below. The consideration of smoking as a covariate in the analyses had only a significant influence on the testosterone results. Thus, all final regression analyses presented below were unadjusted for smoking status, except 
Table 1. Sociodemographic and clinical characteristics of the sample $(N=46)$

\begin{tabular}{|c|c|c|c|c|c|c|c|c|}
\hline & \multicolumn{2}{|c|}{$\begin{array}{l}\text { Healthy controls } \\
(n=25)\end{array}$} & \multicolumn{2}{|c|}{$\begin{array}{l}\text { BPD } \\
(n=21)\end{array}$} & \multirow[t]{2}{*}{$p$ value } & \multirow[t]{2}{*}{$\begin{array}{l}\text { Cohen's } d / \\
\text { Cramer's V }\end{array}$} & \multirow[t]{2}{*}{$95 \% \mathrm{Cl}$} & \\
\hline & $M, n$ & $\mathrm{SD}, \%$ & $M, n$ & $\mathrm{SD}, \%$ & & & & \\
\hline Age & 15.08 & 1.15 & 15.52 & 1.08 & 0.187 & -0.40 & -0.98 & 0.19 \\
\hline BMI & 20.18 & 2.31 & 21.30 & 2.77 & 0.143 & -0.44 & -1.03 & 0.15 \\
\hline School type & & & & & 0.239 & 0.30 & & \\
\hline Hauptschule & 0 & 0 & 1 & 4.8 & & & & \\
\hline Realschule & 7 & 28 & 9 & 42.9 & & & & \\
\hline Gymnasium & 18 & 72 & 10 & 47.6 & & & & \\
\hline Berufsausbildung & 0 & 0 & 1 & 4.8 & & & & \\
\hline German as native language & 25 & 100 & 20 & 95.2 & 0.270 & 0.16 & & \\
\hline Days with physical activity, $n$ & 29.96 & 19.72 & 29.52 & 28.29 & 0.951 & 0.02 & -0.56 & 0.60 \\
\hline Hours of physical activity per day, $n$ & 1.58 & 0.51 & 1.57 & 0.69 & 0.958 & 0.02 & -0.59 & 0.62 \\
\hline Smoker & 0 & 0 & 14 & 66.67 & $<0.001^{1}$ & 0.72 & & \\
\hline Hormonal contraceptives & 5 & 20 & 6 & 28.6 & 0.497 & 0.10 & & \\
\hline Physical illness & 5 & 20 & 7 & 33.3 & 0.305 & 0.15 & & \\
\hline DSM-IV BPD criteria met, $n$ & & & 6.19 & 1.12 & & & & \\
\hline Criterion 1 (abandonment fear) & & & 8 & 38.1 & & & & \\
\hline Criterion 2 (relationship difficulties) & & & 17 & 80.9 & & & & \\
\hline Criterion 3 (identity disturbance) & & & 15 & 71.4 & & & & \\
\hline Criterion 4 (impulsivity) & & & 11 & 52.4 & & & & \\
\hline $\begin{array}{l}\text { Criterion } 5 \text { (suicidal/self-mutilating } \\
\text { behavior) }\end{array}$ & & & 19 & 90.5 & & & & \\
\hline Criterion 6 (affective instability) & & & 21 & 100 & & & & \\
\hline Criterion 7 (emptiness) & & & 19 & 91.5 & & & & \\
\hline Criterion 8 (anger) & & & 12 & 57.1 & & & & \\
\hline Criterion 9 (paranoia/dissociation) & & & 8 & 38.1 & & & & \\
\hline $\mathrm{AQ}$ total & 1.90 & 0.34 & 2.76 & 0.62 & $<0.001^{1}$ & -1.76 & -2.44 & -1.07 \\
\hline AQ anger & 2.18 & 0.46 & 3.20 & 0.71 & $<0.001^{1}$ & -1.72 & -2.40 & -1.03 \\
\hline $\mathrm{AQ}$ physical aggression & 1.48 & 0.50 & 2.17 & 1.03 & $0.009^{1}$ & -0.88 & -1.48 & -0.27 \\
\hline $\mathrm{AQ}$ verbal aggression & 2.28 & 0.47 & 2.68 & 1.03 & $0.116^{1}$ & -0.51 & -1.10 & 0.08 \\
\hline AQ hostility & 1.88 & 0.55 & 3.09 & 0.72 & $<0.001$ & -1.91 & -2.61 & -1.20 \\
\hline BIS-11 total & 63.96 & 8.74 & 77.29 & 13.73 & $0.001^{1}$ & -1.18 & -1.81 & -0.54 \\
\hline BIS-11 attention & 16.48 & 3.43 & 22.52 & 3.08 & $<0.001$ & -1.85 & -2.54 & -1.14 \\
\hline BIS-11 motor & 21.92 & 3.93 & 24.67 & 6.68 & $0.107^{1}$ & -0.51 & -1.10 & 0.08 \\
\hline BIS-11 nonplanning & 25.56 & 3.19 & 30.10 & 6.83 & $0.009^{1}$ & -0.88 & -1.48 & -0.26 \\
\hline YPI total & 1.78 & 0.28 & 2.05 & 0.58 & $0.059^{1}$ & -0.62 & -1.21 & -0.02 \\
\hline YPI Grandiose, manipulative & 1.61 & 0.45 & 1.62 & 0.65 & 0.996 & -0.01 & -0.59 & 0.57 \\
\hline YPI Callous, unemotional & 1.62 & 0.27 & 2.03 & 0.68 & $0.017^{1}$ & -0.81 & -1.41 & -0.20 \\
\hline YPI Impulsive, irresponsible & 2.16 & 0.36 & 2.65 & 0.60 & $0.002^{1}$ & -1.02 & -1.64 & -0.40 \\
\hline
\end{tabular}

${ }^{1} t$ test with unequal variances. AQ, Aggression Questionnaire; BIS-11, Barratt Impulsiveness Scale; BMI, body mass index; BPD, borderline personality disorder; $Y \mathrm{PI}$, Youth Psychopathic Traits Inventory, $\mathrm{Cl}$, confidence interval; $\mathrm{M}$, mean, SD, standard deviation.

for the regression analysis predicting testosterone. As no significant group difference was found in body mass index, physical activity, hormonal contraceptives, or physical illness (see below), these variables were not considered as control variables in the regression analyses. The association between aggressive behavior and cortisol, testosterone, or HR, respectively, was tested by repeating the regression analyses with aggressive behavior (as assessed by the TAP) as an additional predictor variable. Unstandardized effect sizes in the form of differences in means for the main effects and differences-in-differences for the interactions are reported for all outcome variables. Finally, the correlation between cortisol and HR over time was examined using a stepwise mixed-effect regression analysis minimizing the BIC, with log (cortisol) as dependent variable, GROUP and HR (blocks of five minutes each; 0-5, 5-10, and 10-15 min before the saliva sampling) as well as their interaction as fixed effects, and the subject ID as a random effect. In this analysis, log (cortisol) was applied to account for non-normality of residuals. The statistical significance level was set to $\alpha=0.05$. All analyses were conducted using the statistical software Stata 16.0 [61].

\section{Results}

\section{Sample Characteristics}

Sociodemographic and clinical characteristics by group are provided in Table 1. Adolescents with BPD 


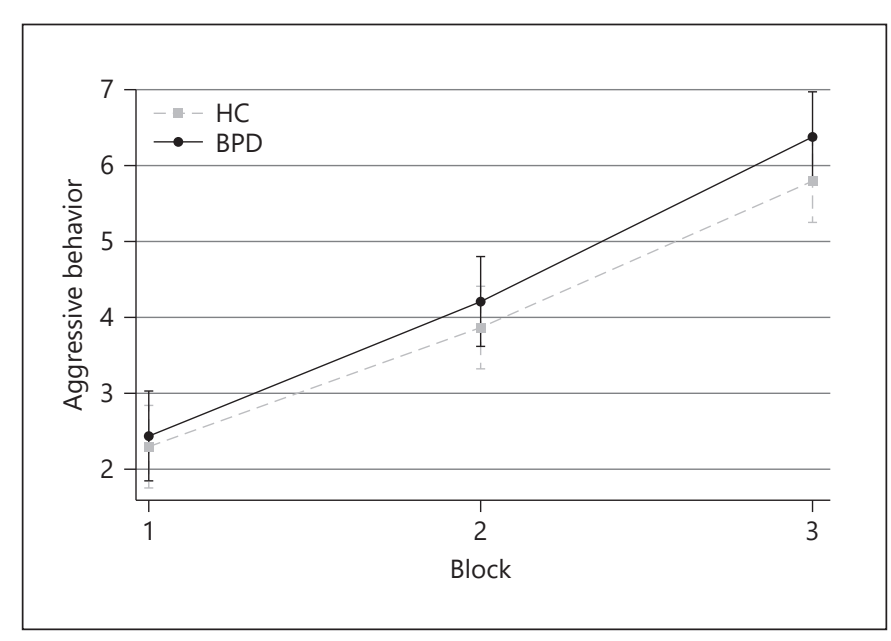

Fig. 2. Aggressive behavior by group and time of measurement. Mean aggressive behavior and 95\% confidence interval over the three blocks of the TAP in the BPD patient group $(n=21)$ and the HC group $(n=25)$. TAP, Taylor Aggression Paradigm; BPD, borderline personality disorder; $\mathrm{HC}$, healthy controls.

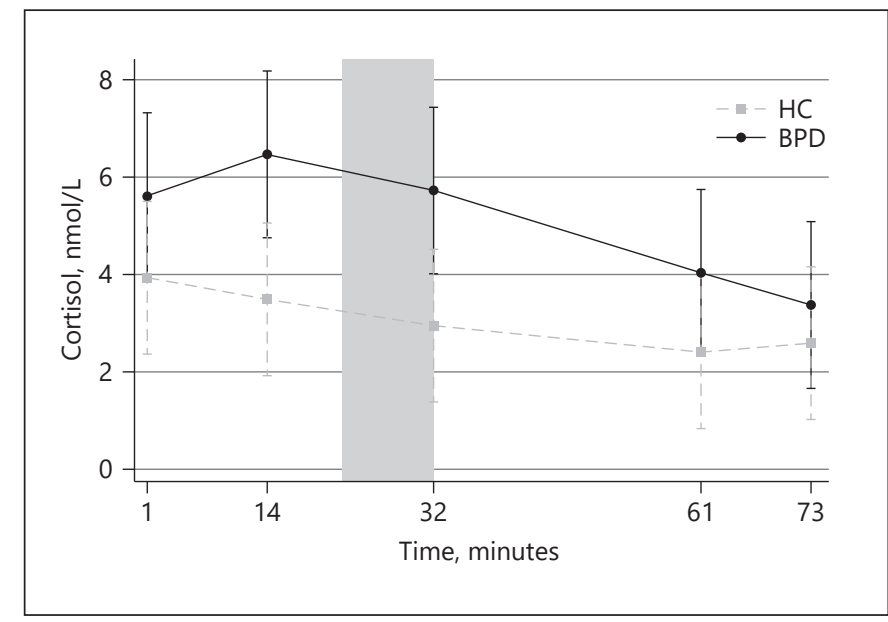

Fig. 3. Endocrinological reactivity by group and time of measurement. Mean cortisol levels including 95\% confidence interval in the BPD patient group $(n=21)$ and the healthy control group $(n=25)$. Times of cortisol measurement were 23 minutes before (T0) and again nine minutes before (T1) the beginning of the TAP, as well as zero minutes (T2), 28 minutes (T3), and 41 minutes (T4) after the end of the TAP. The gray area indicates the time period of the TAP. TAP, Taylor Aggression Paradigm; BPD, borderline personality disorder; HC, healthy controls.

Table 2. Positive and negative affect measured by the PANAS

\begin{tabular}{|c|c|c|c|c|c|c|}
\hline & \multicolumn{2}{|l|}{ Before the TAP } & \multicolumn{2}{|l|}{ After the TAP } & \multicolumn{2}{|c|}{ End of the experimental session } \\
\hline & $\begin{array}{l}\text { healthy controls } \\
(n=25)\end{array}$ & $\begin{array}{l}\text { BPD } \\
(n=21)\end{array}$ & $\begin{array}{l}\text { healthy controls } \\
(n=25)\end{array}$ & $\begin{array}{l}\text { BPD } \\
(n=21)\end{array}$ & $\begin{array}{l}\text { healthy controls } \\
(n=25)\end{array}$ & $\begin{array}{l}\text { BPD } \\
(n=21)\end{array}$ \\
\hline Positive affect & 27.68 (6.09) & $17.57(4.68)$ & $26.52(7.15)$ & $17.00(5.87)$ & $23.20(6.18)$ & $14.76(4.01)$ \\
\hline Negative affect & $13.56(3.61)$ & $18.76(6.44)$ & $14.68(4.78)$ & 19.19 (6.67) & $11.8(2.53)$ & $15.05(6.05)$ \\
\hline
\end{tabular}

Numbers represent means and standard deviations. TAP, Taylor Aggression Paradigm; BPD, borderline personality disorder; PANAS, Positive and Negative Affect Schedule.

were more frequently smokers and scored higher on trait aggression (AQ), impulsivity (BIS-11), and callous-unemotional and impulsive-irresponsible traits (YPI) compared with the healthy control group.

\section{Behavioral Aggression}

Means and standard errors of aggressive behavior for each group and block of the TAP are provided in Figure 2. The regression analysis revealed a significant main effect of $\operatorname{BLOCK}\left(\chi^{2}(2)=964.67, p<0.001\right)$ on aggressive behavior. The main effect of GROUP $\left(\chi^{2}(1)=0.85, p=0.358\right)$ and the interaction of GROUP by BLOCK $\left(\chi^{2}(2)=3.34, p=0.188\right)$ were not significant. The effect sizes are given in the online supplementary Table 1 (for all online suppl. material, see www.karger.com/doi/10.1159/000520228 ). As displayed in Figure 2, adolescents with BPD and healthy controls showed an increase of aggressive behavior from block 1 to block 3 of the TAP, with a nonsignificant group difference in the increase of aggressive behavior. Correlation analyses revealed that aggressive behavior in block 3 was not related to any of the scales of the AQ, BIS-11, and YPI in either group (BPD group: $-0.53<$ all $r>0.41$, all $p>0.064$; healthy control group: $-0.25<$ all $r>0.27$, all $p>0.193$ ).

\section{Affective Reactivity}

The main effects of GROUP and TIME were significant for both PA (GROUP: $\chi^{2}$ (1) 37.22, $p<0.001$; TIME: $\left.\chi^{2}(2)=39.10, p<0.001\right)$ and NA (GROUP: $\chi^{2}(1)=11.11$,
42 


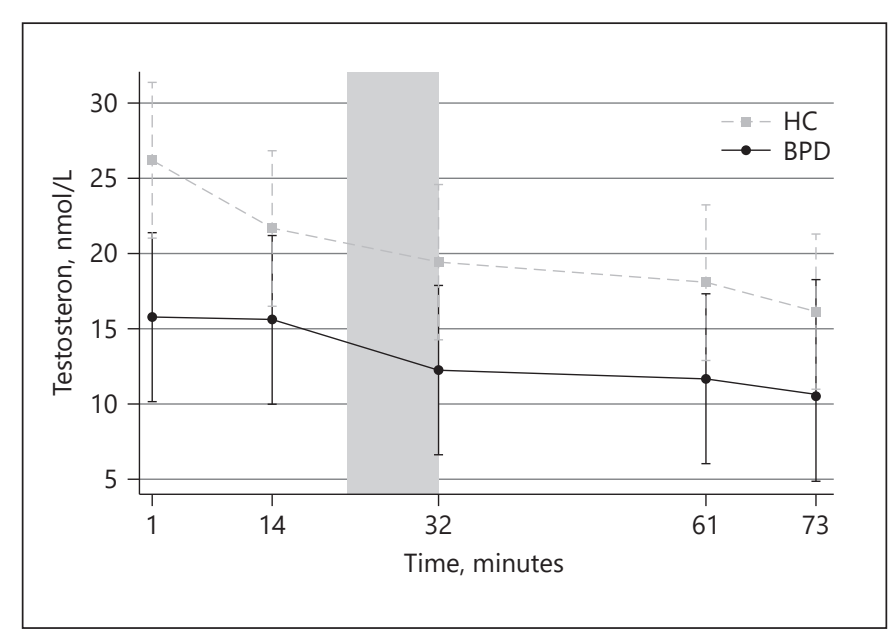

Fig. 4. Testosterone reactivity by group and time of measurement. Mean testosterone levels including 95\% confidence interval in the BPD patient group $(n=21)$ and the healthy control group $(n=25)$. Times of testosterone measurement were 23 minutes before (T0) and again nine minutes before (T1) the beginning of the TAP, as well as zero minutes (T2), 28 minutes (T3), and 41 minutes (T4) after the end of the TAP. The gray area indicates the time period of the TAP. TAP, Taylor Aggression Paradigm; BPD, borderline personality disorder; HC, healthy controls.

$p<0.001$; TIME: $\left.\chi^{2}(2)=34.84, p<0.001\right)$. The GROUP by TIME interaction was neither significant for PA $\left(\chi^{2}(2)\right.$ $=1.94, p=0.380)$ nor for NA $\left(\chi^{2}(2)=2.51, p=0.285\right)$. Table 2 presents the means and standard deviations for PA and NA separated by group, and in the online supplementary Table 1 shows the effect sizes. There was no significant change in PA between before and after the TAP $\left(\chi^{2}(1)=2.02, p=0.155\right)$, but PA significantly decreased between after the TAP and the end of the experimental session $\left(\chi^{2}(1)=20.82, p<0.001\right)$. Similarly, there was no significant change in NA between before and after the TAP $\left(\chi^{2}(1)=1.53, p=0.216\right)$, but NA significantly decreased between after the TAP and the end of the experimental session $\left(\chi^{2}(1)=31.55, p<0.001\right)$.

\section{Cortisol Reactivity}

Mean and 95\% CI for cortisol levels are displayed in Figure 3. The regression analysis revealed a significant main effect of TIME on the cortisol response $\left(\chi^{2}(4)=\right.$ $66.23, p<0.001)$, while the main effect of GROUP was not significant $\left(\chi^{2}(1)=3.13, p=0.077\right)$. The GROUP by TIME interaction on the cortisol response was significant $\left(\chi^{2}(4)=16.49, p=0.002\right)$. See in the online supplementary Table 1 for the effect sizes. As displayed in Figure 3, adolescents with BPD showed an increase in cortisol from baseline (T0) to prior to the TAP (T1), followed by a con-

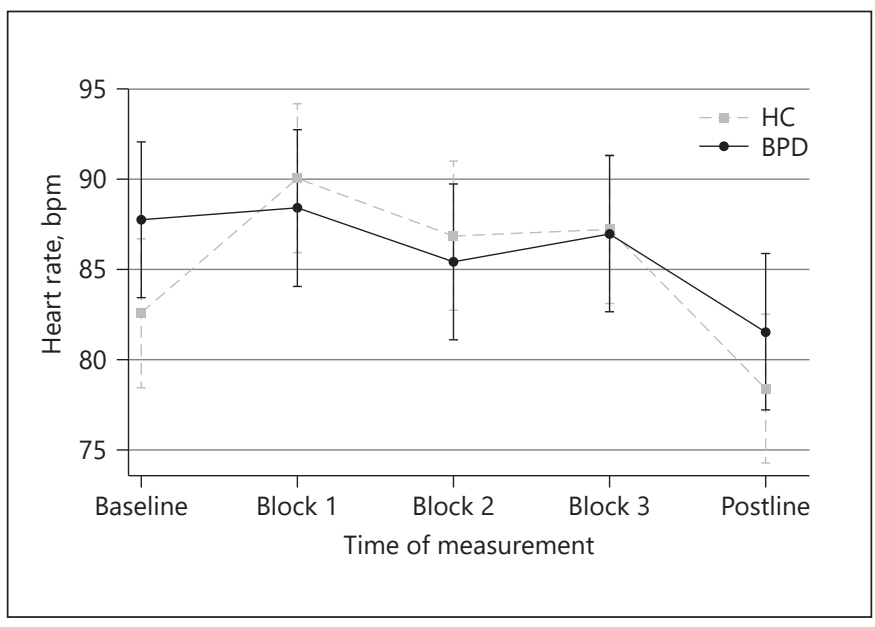

Fig. 5. Autonomic reactivity by group and time of measurement. Mean HR including the $95 \%$ confidence interval in the BPD patient group $(n=21)$ and the healthy control group $(n=25)$. HR was measured in 5-min intervals during resting conditions at baseline and postline, and individually during the TAP (block 1-3). TAP, Taylor Aggression Paradigm; BPD, borderline personality disorder; HR, heart rate; HC, healthy controls.

tinuous decrease in cortisol toward the end of the experimental session (T4). In contrast, healthy controls demonstrated a decrease in cortisol from the beginning (T0) to the end of the experimental session (T4). A significant group difference in the change of cortisol levels over time was only found between baseline (T0) and prior to the TAP $(\mathrm{T} 1)\left(\chi^{2}(1)=4.28, p=0.039\right)$. When the regression analysis was repeated with aggressive behavior (as assessed by the TAP) as an additional predictor variable, the association between aggressive behavior and cortisol was not significant $(B=0.56, p=0.057)$.

\section{Testosterone Reactivity}

Mean and 95\% CI for testosterone levels are displayed in Figure 4 . The regression analysis revealed a significant main effect of TIME on the testosterone response $\left(\chi^{2}(4)=42.29\right.$, $p<0.001)$, while neither the main effect of GROUP $\left(\chi^{2}(1)\right.$ $=1.77, p=0.184)$ nor the GROUP by TIME interaction $\left(\chi^{2}\right.$ $(4)=4.18, p=0.383)$ on the testosterone response was significant. The effect sizes are given in in the online supplementary Table 1. As displayed in Figure 4, both groups showed a continuous decrease in testosterone from the beginning (T0) to the end of the experimental session (T4). When the regression analysis was repeated with aggressive behavior (as assessed by the TAP) as an additional predictor variable, the association between aggressive behavior and testosterone was not significant $(B=-1.51, p=0.110)$. 


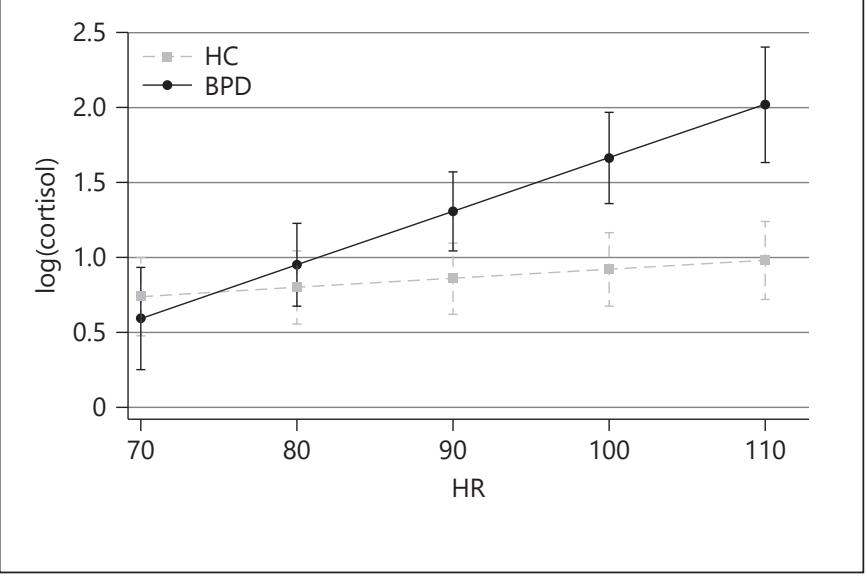

Fig. 6. Relationship between $\log$ (cortisol) and HR by group and time of measurement. $\mathrm{HR}$, heart rate; $\mathrm{BPD}$, borderline personality disorder; HC, healthy controls.

\section{Autonomic Reactivity}

Mean and 95\% CI for HR are displayed in Figure 5. The regression analysis revealed a significant effect of BLOCK on HR $\left(\chi^{2}(4)=65.70, p<0.001\right)$, while the main effect of GROUP was not significant $\left(\chi^{2}(1)=0.14\right.$, $p=0.705)$. The GROUP by BLOCK interaction on HR was significant $\left(\chi^{2}(4)=12.66, p<0.013\right)$. See in the online supplementary Table 1 for the effect sizes. As displayed in Figure 5, healthy controls showed a significantly greater increase in HR from baseline to block 1 of the TAP $\left(\chi^{2}(1)=8.04, p=0.005\right)$ compared with adolescents with BPD. Both groups demonstrated a decrease of HR from block 1 toward the end of the experimental session, with no significant group differences in changes of HR between the remaining measurement points. When the regression analysis was repeated with aggressive behavior (as assessed by the TAP) as an additional predictor variable, the association between aggressive behavior and HR was significant $(B=1.57, p=$ $0.001)$.

\section{Association between the Endocrinological and Autonomic Reactivity}

Log (cortisol) was significantly predicted with mean HR (5-min blocks) 10-15 min before the saliva sampling for both the healthy control group $(\beta=0.14, p=0.031)$ as well as for the BPD group $(\beta=0.86, p<0.001)$. As shown in Figure 6, the association between cortisol and HR was stronger for the BPD group compared with the healthy control group $(z=4.26, p<0.001)$.

\section{Discussion}

The present study applied the TAP to examine aggressive behavior and its biological correlates in female adolescents with BPD compared to healthy controls. The following main findings emerged from the study: First, while both groups behaved increasingly aggressive over the three blocks of the TAP, the aggressive behavior in the BPD group was not stronger than in the healthy control group, and self-reports of trait aggression and impulsivity were unrelated to aggressive behavior. Second, the TAP did not trigger a subjective affective reaction either in the BPD group or the healthy control group. Third, the expected cortisol and testosterone increases due to the aggression induction through the TAP were not observed in either group, and neither cortisol nor testosterone was correlated with aggressive behavior. Instead, we found an increase in cortisol in the time preceding the TAP $(-23$ $\min$ to $-9 \mathrm{~min}$ ) in the BPD group only. Fourth, there was no HR increase due to the accelerating aggression induction over the three blocks of the TAP in either group, even though HR was positively correlated with aggressive behavior. Instead, we found a significantly greater HR increase from baseline to the first block of the TAP in the healthy control group compared to the BPD group. Finally, we found a positive association between HR and the cortisol level 10-15 minutes later, which was more pronounced in adolescents with BPD compared to healthy controls.

In the current study, adolescents with BPD did not behave more aggressively than healthy controls. These results are in contrast to the literature suggesting that individuals with BPD are susceptible to aggressive behavior $[7,8]$. A possible explanation for the nonsignificant group difference in our study is that the aggression provocation in the TAP did not trigger the specific mechanisms underlying aggressive behavior in individuals with BPD, such as hypersensitivity to interpersonal rejection [10] or invalidation (i.e., the rejection of one's emotional experience) [62]. This is in line with the criticism that aggressive behaviors within laboratory-based paradigms are contrived and are nothing like typical social behaviors in participants' day-to-day lives $[63,64]$. Additionally, the null finding for the group difference in aggressive behavior could also be explained by the heterogeneity of the disorder [65]. Only a subgroup of participants in the current study met the DSM-IV criterion for anger $(57 \%)$ or impulsivity (52\%) that were found to be associated with aggressive behavior in young people with BPD pathology [17]. The small sample size in the current study prevented
44

Psychopathology 2022;55:37-48 DOI: $10.1159 / 000520228$
Cavelti et al. 
subgroup analyses. However, this should be focussed in future research.

In contrast to our hypothesis, neither the BPD group nor the HC group showed a significant change in affect between before and after the TAP. Instead, both groups reported greater PA and NA at baseline compared with the end of the experimental session, with no group difference in the decrease of PA and NA over time. This unexpected finding stands in contrast to recent findings [7, 45 ], and questions that the TAP in our study led to affective responses. It may have further contributed to the nonsignificant difference in aggressive behavior between the BPD group and the healthy control group, as the failure to regulate negative feelings has been suggested to lead individuals with BPD to respond more aggressively [7]. A potential explanation could be that the participants showed a high level of affective arousal at the beginning of the experimental session, because they were excited in anticipation of the upcoming experiment, which slowly subsided over the course of the experiment.

The null-findings for testosterone in the current study are in line with previous studies reporting no testosterone response on the aggression induction through the TAP in healthy, female adolescents [50], and no relation between testosterone and trait anger or aggressiveness in a gendermixed sample of adults with BPD [37].

While there were no group differences in cortisol and HR responses to the aggression induction through the TAP, the BPD group and the healthy control group did differ in their cortisol and HR responses during the preparatory phase prior the actual encounter with the experimental task. The elevated cortisol levels at the beginning of the experimental session in adolescents with BPD compared to the $\mathrm{HC}$ group are in line with the meta-analysis by Drews et al. [35], but contradict the meta-analysis by Thomas et al. [34]. The observed cortisol increase in the time preceding the TAP in adolescents with BPD could be interpreted as the result of fearful anticipation of the potentially threatening interaction with another person during the experiment [66], as the TAP comprises punishment within an interpersonal competition that might activate the brain's threat response system [67]. In contrast, healthy controls demonstrated a HR increase from baseline to the start of the TAP, which can be considered as an adaptive response of the stress regulatory system that allows individuals to make behavioral, cognitive, and physiological adjustments that are necessary to deal with the upcoming requirements. This interpretation is in line with research demonstrating that patients with BPD show increased threat sensitivity that is linked

Aggression in Adolescents with BPD to their aggressiveness [68], and that disturbed emotion processing in BPD does not only occur during the perception of emotional stimuli, but also during the anticipation of upcoming emotional stimuli [69]. Additionally, it corresponds with the claim that some laboratory-based aggression paradigms supposedly measuring aggression actually operationalize competitiveness [63].

The finding of a positive association between HR and cortisol levels that was more pronounced in the BPD group is new and requires further explanation. There is evidence from studies in healthy adults suggesting an inverse relationship between cortisol levels and the activity of the vagus nerve (e.g., indexed by HRV), the main component of the parasympathetic division of the ANS, which could reflect the inhibitory influence of the prefrontal cortex on the amygdala, which initiates the HPA axis response to stress [70, 71]. Interestingly, Pulopulos et al. [71] found that a larger decrease in HRV during the anticipation of a stress task (reflecting poorer stress/emotion regulation associated with reduced prefrontal activity) was related to higher stress task-induced cortisol increase, while the change in HRV specifically related to the stress task was not associated with differences in the HPA axis response to stress. In light of these findings, the positive association between HR and cortisol changes in the current study may indicate that the inhibitory effect of the vagus nerve on the HPA axis activity in the BPD group is impaired. More research is needed to replicate this finding and to examine in more detail how individuals with BPD differ from healthy controls, concerning physiological changes during the anticipation of a stressful event in distinction to those due to the aggression induction itself.

Several limitations of the current study should be considered. First, the sample was small. As a result, the power to detect an effect in the current study, if there was a real effect to be detected, was rather low, particularly with regard to the interaction effects (see in online supplementary Table 2 for the estimated minimal effect sizes the current study was powered to detect). Second, the sample consisted exclusively of females, because the majority of individuals with BPD in the clinical setting are female, and it allowed us to control for the influence of sex on the biological responses [72]. However, it limits the generalizability of our findings. Third, as healthy controls were used as the control group, we cannot rule out that some of the findings may be associated with psychopathology in general rather than with BPD per se. Future research should apply a dimensional approach to assess BPD pathology. Fourth, we did not control for pubertal stage and age of menarche onset that have been shown to influence 
hormone levels and ANS function. Fifth, assessing participants during their follicular phase of the menstrual cycle may have dampened their aggressive response in the $\mathrm{TAP}$, as preliminary research suggests that greater aggression occurs in the mid-to late-luteal phase [73]. Sixth, baseline group differences in cortisol and HR were observed that may have influenced the results. Future research would do well to implement a habituation phase before the actual start of the experiment which would allow to better disentangle physiological stress effects related to the general experimental procedures from those related to the aggression induction per se. Seventh, due to technical restrictions of the electrodes used we were not able to measure HRV that has been found to be associated with BPD pathology $[40,41,43]$. Finally, as the current study applied a modified version of the TAP that uses acoustic tone blasts [44-46] instead of electric shocks [44] or a monetary provocation [7], it cannot be ruled out that the failure to replicate previous results is due to the respective modification to the TAP.

To conclude, in the current study we found no evidence of increased aggression in adolescents with BPD compared with healthy controls, either at the phenomenological or the biological level. Our results suggest that adolescents with BPD differ from healthy controls in the anticipation of the experimental task that initiates biological changes to facilitate the confrontation with the upcoming stressor. Future studies are needed to further examine the validity of this observation and its interpretation.

\section{Acknowledgments}

We thank all participants who were willing to take part in the study.

\section{Statement of Ethics}

The study was approved by the Ethics Committee of the Medical Faculty at the University of Heidelberg, Germany (Study-ID: S-630/2013), and was conducted in accordance with the declaration of Helsinki [52]. Written informed consent was obtained from all participants and their parents or legal guardians.

\section{Conflict of Interest Statement}

The authors have no conflicts of interest to declare.

\section{Funding Sources}

The study was funded by the Competence Center of Prevention, Diagnostic and Therapy of Aggressive Disorders in Child and Adolescent innovation fund medicine of the State of Baden-Württemberg, Germany. The foundation had no involvement in the collection, analysis, or interpretation of data, in the writing of the manuscript, or the decision to submit the article for publication.

\section{Author Contributions}

M.K. developed the study idea and design. L.R., P.P., J.K., K.B., R.B., F.R., and M.K. took an active part in the study implementation, recruitment, and data management. L.R. collected the data. M.C., P.P., and J.J. analyzed the data. M.C. wrote the first draft of the manuscript. All authors contributed to and approved the current version of the manuscript.

\section{Data Availability Statement}

The data are available from the corresponding author on request.

\section{References}

1 Gunderson JG, Herpertz SC, Skodol AE, Torgersen S, Zanarini MC. Borderline personality disorder. Nat Rev Dis Primers. 2018 May;4:18029.

2 Sharp C, Fonagy P. Practitioner review: borderline personality disorder in adolescence - recent conceptualization, intervention, and implications for clinical practice. J Child Psychol Psychiatry. 2015 Dec;56(12): 1266-88.

3 Mancke F, Herpertz SC, Bertsch K. Correlates of aggression in personality disorders: an update. Curr Psychiatry Rep. 2018 Aug; 20(8):53.
4 Peters JR, Derefinko KJ, Lynam DR. Negative urgency accounts for the association between borderline personality features and intimate partner violence in young men. J Pers Disord. 2017;31(1):16-25.

5 González RA, Igoumenou A, Kallis C, Coid JW. Borderline personality disorder and violence in the UK population: categorical and dimensional trait assessment. BMC Psychiatry. 2016 03;16:180.

6 Armenti NA, Babcock JC. Borderline personality features, anger, and intimate partner violence: an experimental manipulation of rejection. J Interpers Violence. 2018 Apr;36(5-6): NP3104-29.
7 Kogan-Goloborodko O, Brügmann E, Repple J, Habel U, Clemens B. Experimentally assessed reactive aggression in borderline personality disorder. PLoS One. 2016 Nov; 11(11): 0166737.

8 Mancke F, Herpertz SC, Bertsch K. Aggression in borderline personality disorder: a multidimensional model. Personal Disord. 2015;6(3):278-91.

9 Newhill CE, Eack SM, Mulvey EP. A growth curve analysis of emotion dysregulation as a mediator for violence in individuals with and without borderline personality disorder. J Pers Disord. 2012 Jun;26(3):452-67. 
10 Scott LN, Wright AGC, Beeney JE, Lazarus SA, Pilkonis PA, Stepp SD. Borderline personality disorder symptoms and aggression: a within-person process model. J Abnorm Psychol. 2017 May;126(4):429-40.

11 Scott LN, Stepp SD, Pilkonis PA. Prospective associations between features of borderline personality disorder, emotion dysregulation, and aggression. Personal Disord. 2014 Jul; 5(3):278-88.

12 van Asselt AD, Dirksen CD, Arntz A, Severens JL. The cost of borderline personality disorder: societal cost of illness in BPD-patients. Eur Psychiatry. 2007 Sep;22(6):354-61.

13 Moore KE, Tull MT, Gratz KL. Borderline personality disorder symptoms and criminal justice system involvement: the roles of emotion-driven difficulties controlling impulsive behaviors and physical aggression. Compr Psychiatry. 2017;76:26-35.

14 Johnson JG, Cohen P, Smailes E, Kasen S, Oldham JM, Skodol AE, et al. Adolescent personality disorders associated with violence and criminal behavior during adolescence and early adulthood. Am J Psychiatry. 2000 Sep;157(9):1406-12.

15 Arola R, Antila H, Riipinen P, Hakko H, Riala K, Kantojärvi L. Borderline personality disorder associates with violent criminality in women: a population based follow-up study of adolescent psychiatric inpatients in Northern Finland. Forensic Sci Int. 2016 Sep;266: 389-95.

16 Robitaille MP, Checknita D, Vitaro F, Tremblay RE, Paris J, Hodgins S. A prospective, longitudinal, study of men with borderline personality disorder with and without comorbid antisocial personality disorder. Borderline Personal Disord Emot Dysregul. 2017;4:25.

17 Cavelti M, Thompson K, Betts J, Fowler C, Luebbers S, Cotton SM, et al. Borderline personality disorder diagnosis and symptoms in outpatient youth as risk factors for criminal offences and interpersonal violence. J Personal Disord. 2021 Jan:1-24. Accepted.

18 Barzman DH, Patel A, Sonnier L, Strawn JR. Neuroendocrine aspects of pediatric aggression: can hormone measures be clinically useful? Neuropsychiatr Dis Treat. 2010 Oct;6: 691-7.

19 Fragkaki I, Cima M, Granic I. The role of trau$\mathrm{ma}$ in the hormonal interplay of cortisol, testosterone, and oxytocin in adolescent aggression. Psychoneuroendocrinology. 2018 Feb; 88:24-37.

20 Hawes DJ, Brennan J, Dadds MR. Cortisol, callous-unemotional traits, and pathways to antisocial behavior. Curr Opin Psychiatry. 2009 Jul;22(4):357-62.

21 Platje E, Jansen LM, Raine A, Branje SJ, Doreleijers TA, de Vries-Bouw M, et al. Longitudinal associations in adolescence between cortisol and persistent aggressive or rule-breaking behavior. Biol Psychol. 2013 Apr;93(1):1327.

22 Stoppelbein L, Greening L, Luebbe A, Fite P, Becker SP. The role of cortisol and psycho- pathic traits in aggression among at-risk girls: tests of mediating hypotheses: aggression among girls. Aggress Behav. 2014 May;40(3): 263-72.

23 Shoal GD, Giancola PR, Kirillova GP. Salivary cortisol, personality, and aggressive behavior in adolescent boys: a 5-Year Longitudinal Study. J Am Acad Child Adolesc Psychiatry. 2003 Sep;42(9):1101-7.

24 Duke SA, Balzer BW, Steinbeck KS. Testosterone and Its effects on human male adolescent mood and behavior: a systematic review. J Adolesc Health. 2014 Sep;55(3):315-22.

25 Yildirim BO, Derksen JJ. A review on the relationship between testosterone and lifecourse persistent antisocial behavior. Psychiatry Res. 2012 Dec;200(2-3):984-1010.

26 Platje E, Popma A, Vermeiren RRJM, Doreleijers TAH, Meeus WHJ, van Lier PAC, et al. Testosterone and cortisol in relation to aggression in a non-clinical sample of boys and girls: testosterone and cortisol associations with aggression. Aggress Behav. 2015 Sep; 41(5):478-87.

27 Ortiz J, Raine A. Heart rate level and antisocial behavior in children and adolescents: a meta-analysis. J Am Acad Child Adolesc Psychiatry. 2004 Feb;43(2):154-62.

28 Raine A, Fung ALC, Portnoy J, Choy O, Spring VL. Low heart rate as a risk factor for child and adolescent proactive aggressive and impulsive psychopathic behavior: low heart rate, aggression, and child psychopathy. Aggress Behav. 2014 Jul;40(4):290-9.

29 Fagan SE, Zhang W, Gao Y. Social adversity and antisocial behavior: mediating effects of autonomic nervous system activity. J Abnorm Child Psychol. 2017 Nov;45(8):155364.

30 Sijtsema JJ, Veenstra R, Lindenberg S, van Roon AM, Verhulst FC, Ormel J, et al. Mediation of sensation seeking and behavioral inhibition on the relationship between heart rate and antisocial behavior: the TRAILS Study. J Am Acad Child Adolesc Psychiatry. 2010 May;49(5):493-502.

31 Baker LA, Tuvblad C, Reynolds C, Zheng M, Lozano DI, Raine A. Resting heart rate and the development of antisocial behavior from age 9 to 14: genetic and environmental influences. Dev Psychopathol. 2009 Aug;21(3): 939-60.

32 Portnoy J, Farrington DP. Resting heart rate and antisocial behavior: an updated systematic review and meta-analysis. Aggress Violent Behav. 2015 May;22:33-45.

33 Portnoy J, Raine A, Chen FR, Pardini D, Loeber R, Jennings JR. Heart rate and antisocial behavior: the mediating role of impulsive sensation seeking. Criminology. 2014 May;52(2): 292-311.

34 Thomas N, Gurvich C, Hudaib AR, Gavrilidis E, Kulkarni J. Systematic review and metaanalysis of basal cortisol levels in borderline personality disorder compared to non-psychiatric controls. Psychoneuroendocrinology. 2019 Apr;102:149-57.
35 Drews E, Fertuck EA, Koenig J, Kaess M, Arntz A. Hypothalamic-pituitary-adrenal axis functioning in borderline personality disorder: a meta-analysis. Neurosci Biobehav Rev. 2019 Jan;96:316-34.

36 Bertsch K, Roelofs K, Roch PJ, Ma B, Hensel S, Herpertz SC, et al. Neural correlates of emotional action control in anger-prone women with borderline personality disorder. J Psychiatry Neurosci. 2018 May;43(3): 170102-70.

37 Rausch J, Gäbel A, Nagy K, Kleindienst N, Herpertz SC, Bertsch K. Increased testosterone levels and cortisol awakening responses in patients with borderline personality disorder: gender and trait aggressiveness matter. Psychoneuroendocrinology. 2015 May;55: $116-27$

38 Dettenborn L, Kirschbaum C, Gao W, Spitzer C, Roepke S, Otte C, et al. Increased hair testosterone but unaltered hair cortisol in female patients with borderline personality disorder. Psychoneuroendocrinology. 2016 Sep; 71: 176-9.

39 Deuter CE, Duesenberg M, Hellmann-Regen J, Metz S, Roepke S, Wolf OT, et al. Psychosocial stress increases testosterone in patients with borderline personality disorder, posttraumatic stress disorder and healthy participants. Bord Personal Disord Emot Dysregul. 2021 Dec;8(1):3

40 Koenig J, Kemp AH, Feeling NR, Thayer JF, Kaess M. Resting state vagal tone in borderline personality disorder: a meta-analysis. Prog Neuropsychopharmacol Biol Psychiatry. 2016 Jan;64:18-26.

41 Koenig J, Rinnewitz L, Parzer P, Resch F, Thayer JF, Kaess M. Resting cardiac function in adolescent non-suicidal self-injury: the impact of borderline personality disorder symptoms and psychosocial functioning. Psychiatry Res. 2017 Feb;248:117-20.

42 Weise S, Parzer P, Zimmermann R, Fürer L, Resch F, Kaess M, et al. Emotion dysregulation and resting-state autonomic function in adolescent borderline personality disorder: a multimodal assessment approach. Personal Disord. 2020 Jan;11(1):46-53.

43 Koenig J, Weise S, Rinnewitz L, Parzer P, Resch F, Kaess M. Longitudinal covariance of resting-state cardiac function and borderline personality disorder symptoms in adolescent non-suicidal self-injury. World J Biol Psychiatry. 2018 Feb;19(2):152-7.

44 Taylor SP. Aggressive behavior and physiological arousal as a function of provocation and the tendency to inhibit aggression. J Pers. 1967 Jun;35(2):297-310.

45 Böhnke R, Bertsch K, Kruk MR, Naumann E. The relationship between basal and acute HPA axis activity and aggressive behavior in adults. J Neural Transm. 2010 May;117(5):629-37.

46 Böhnke R, Bertsch K, Kruk MR, Richter S, Naumann E. Exogenous cortisol enhances aggressive behavior in females, but not in males. Psychoneuroendocrinology. 2010 Aug;35(7): 1034-44. 
47 Assaad JM, Pihl RO, Séguin JR, Nagin DS, Vitaro F, Tremblay RE. Intoxicated behavioral disinhibition and the heart rate response to alcohol. Exp Clin Psychopharmacol. 2006; 14(5):377-88.

48 Wagels L, Votinov M, Kellermann T, Konzok J, Jung S, Montag C, et al. Exogenous testosterone and the monoamine-oxidase a polymorphism influence anger, aggression and neural responses to provocation in males. Neuropharmacology. 2019 Jan;156:107491.

49 Berman M, Gladue B, Taylor S. The effects of hormones, Type A behavior pattern, and provocation on aggression in men. Motiv Emot. 1993 Jun;17(2):125-38.

50 Rinnewitz L, Parzer P, Koenig J, Bertsch K, Brunner R, Resch F, et al. A biobehavioral validation of the Taylor aggression paradigm in female adolescents. Sci Rep. 2019 Dec;9(1): 7036.

51 Kaess M, Ghinea D, Fischer-Waldschmidt G Resch F. Die Ambulanz für Risikoverhalten und Selbstschädigung (AtR!Sk): ein Pionierkonzept der ambulanten Früherkennung und Frühintervention von BorderlinePersönlichkeitsstörungen. Prax Kinderpsychol Kinderpsychiatr. 2017 Jul;66(6):404-22.

52 World Medical Association. World Medical Association Declaration of Helsinki: ethical principles for medical research involving human subjects. JAMA. 2013 Nov;310(20) 2191-4.

53 First M, Spitzer R, Gibbon M, Williams J. Structured clinical interview for DSM-IV-TR Axis I disorders research version, non-patient edition (SCID-I/NP). New York: Biometrics Research; 2002.

54 First M, Spitzer R, Gibbon M, Williams J, Benjamin L. Structured clinical interview for DSM-IV Axis II personality disorders (SCIDII). New York: Biometric Research Department; 1994
55 Krohne HW, Egloff B, Kohlmann C-W, Tausch A. Investigations with a German version of the positive and negative affect schedule (PANAS). Diagnostica. 1996;42:139-56.

56 Buss AH, Perry M. The aggression questionnaire. J Pers Soc Psychol. 1992 Sep;63(3):4529.

57 Patton JH, Stanford MS, Barratt ES. Factor structure of the Barratt impulsiveness scale. J Clin Psychol. 1995 Nov;51(6):768-74.

58 Andershed H, Kerr M, Stattin H, Levander S. Psychopathic traits in non-referred youths: a new assessment tool. In: Blaauw E, Sherridan L, editors. Psychopaths: current International perspectives. The Hague: Elsevier; 2002. p. $131-58$.

59 Schneider W, Eschman A, Zuccolotto A. EPrie (version 2.0). Pittsburgh, PA: Psychology Software Tools Inc.; 2002.

60 Shirtcliff EA, Granger DA, Schwartz E, Curran MJ. Use of salivary biomarkers in biobehavioral research: cotton-based sample collection methods can interfere with salivary immunoassay results. Psychoneuroendocrinology. 2001 Feb;26(2):165-73.

61 StataCorp. Stata statistical software: release. Vol. 16. College Station, TX: StataCorp LLC; 2019.

62 Herr NR, Jones AC, Cohn DM, Weber DM. The impact of validation and invalidation on aggression in individuals with emotion regulation difficulties. Personal Disord. 2015 Oct; 6(4):310-4.

63 McCarthy RJ, Elson M. A conceptual review of lab-based aggression paradigms. Collabra Psychol. 2018 Jan;4(1):4.

64 Ritter D, Eslea M. Hot Sauce, toy guns, and graffiti: a critical account of current laboratory aggression paradigms. Aggr. Behav. 2005 Oct;31(5):407-19.

65 Cavelti M, Lerch S, Ghinea D, Fischer-Waldschmidt G, Resch F, Koenig J, et al. Heterogeneity of borderline personality disorder symptoms in help-seeking adolescents. Borderline Personal Disord Emot Dysregul. 2021 Dec;8(1):9.
66 Lyons-Ruth K, Choi-Kain L, Pechtel P, Bertha E, Gunderson J. Perceived parental protection and cortisol responses among young females with borderline personality disorder and controls. Psychiatry Res. 2011 Oct;189(3):42632.

67 Bertsch K, Florange J, Herpertz SC. Understanding brain mechanisms of reactive aggression. Curr Psychiatry Rep. 2020 Dec; 22(12):81.

68 Bertsch K, Krauch M, Stopfer K, Haeussler K, Herpertz SC, Gamer M. Interpersonal threat sensitivity in borderline personality disorder: an Eye-Tracking Study. J Pers Disord. 2017 Oct;31(5):647-70.

69 Scherpiet S, Brühl AB, Opialla S, Roth L, Jäncke L, Herwig U. Altered emotion processing circuits during the anticipation of emotional stimuli in women with borderline personality disorder. Eur Arch Psychiatry Clin Neurosci. 2014 Feb;264(1):45-60.

70 Weber CS, Thayer JF, Rudat M, Wirtz PH, Zimmermann-Viehoff $\mathrm{F}$, Thomas A, et al. Low vagal tone is associated with impaired post stress recovery of cardiovascular, endocrine, and immune markers. Eur J Appl Physiol. 2010 May;109(2):201-11.

71 Pulopulos MM, Vanderhasselt MA, De Raedt R. Association between changes in heart rate variability during the anticipation of a stressful situation and the stress-induced cortisol response. Psychoneuroendocrinology. 2018 Aug;94:63-71.

72 Zorn JV, Schür RR, Boks MP, Kahn RS, Joëls $\mathrm{M}$, Vinkers $\mathrm{CH}$. Cortisol stress reactivity across psychiatric disorders: a systematic review and meta-analysis. Psychoneuroendocrinology. 2017 Mar;77:25-36.

73 Peters JR, Owens SA, Schmalenberger KM, Eisenlohr-Moul TA. Differential effects of the menstrual cycle on reactive and proactive aggression in borderline personality disorder. Aggress Behav. 2020 Mar;46(2):151-61. 